\title{
Dietary administration of resistant starch improved caecal barrier function by enhancing intestinal morphology and modulating microbiota composition in meat duck
}

\author{
Simeng Qin ${ }^{1}$, Keying Zhang ${ }^{1}$, Todd J. Applegate ${ }^{2}$, Xuemei Ding ${ }^{1}$, Shiping Bai ${ }^{1}$, Yuheng Luo ${ }^{1}$, \\ Jianping Wang ${ }^{1}$, Huanwei Peng ${ }^{1}$, Zhuowei $\mathrm{Su}^{1}$, Yue Xuan ${ }^{1}$ and Qiufeng Zeng ${ }^{1 *}$ \\ ${ }^{1}$ Institute of Animal Nutrition, Key Laboratory of Animal Disease-Resistance Nutrition, Ministry of Education, Sichuan \\ Agricultural University, Chengdu, Sichuan 611130, People's Republic of China \\ ${ }^{2}$ Department of Poultry Science, University of Georgia, Athens, GA 30602, USA \\ (Submitted 25 March 2019 - Final revision received 15 August 2019 - Accepted 27 August 2019 - First published online 15 November 2019)
}

\section{Abstract}

Resistant starch (RS) was recently approved to exert a powerful influence on gut health, but the effect of RS on the caecal barrier function in meat ducks has not been well defined. Thus, the effect of raw potato starch (RPS), a widely adopted RS material, on microbial composition and barrier function of caecum for meat ducks was determined. A total of 360 Cherry Valley male ducks of 1-d-old were randomly divided and fed diets with 0 (control), 12, or $24 \%$ RPS for 35 d. Diets supplemented with RPS significantly elevated villus height and villus height:crypt depth ratio in the caecum. The $16 \mathrm{~S}$ rRNA sequence analysis indicated that the diet with $12 \%$ RPS had a higher relative abundance of Firmicutes and the butyrateproducing bacteria Faecalibacterium, Subdoligranulum, and Erysipelatoclostridium were enriched in all diets. Lactobacillus and Bifidobacterium were significantly increased in the $24 \%$ RPS diet $v$. the control diet. When compared with the control diet, the diet with $12 \%$ RPS was also found to notably increase acetate, propionate and butyrate contents and up-regulated barrier-related genes including claudin-1, zonula occludens-1, mucin- 2 and proglucagon in the caecum. Furthermore, the addition of $12 \%$ RPS significantly reduced plasma TNF- $\alpha$, IL- $1 \beta$ and endotoxin concentrations. These data revealed that diets supplemented with $12 \%$ RPS partially improved caecal barrier function in meat ducks by enhancing intestinal morphology and barrier markers expression, modulating the microbiota composition and attenuating inflammatory markers.

Key words: Resistant starch: Caecal microbiota: Barrier function: SCFA: Meat ducks

China is the world's largest contributor to duck production, accounting for three-fourth of the global duck husbandry. Meat ducks are highly sensitive to pathogens and toxins in feed, such as antinutritional factors as well as environmental stress, such as heat and immune stress ${ }^{(1-3)}$. Research has verified that toxins and stresses usually lead to the damage of intestinal morphology and an increased inflammatory response ${ }^{(4,5)}$, which generally result in the occurrence of low-grade inflammation of the intestine in ducks in the production field. The general growth and well-being of animals are related to the health status of the intestine ${ }^{(6)}$. Thus, it is still a big challenge for poultry nutritionists to maintain the gut health of ducks through nutritional strategy.

The intestinal epithelium is part of the mucosa layer that forms a physical barrier to allow the absorption of nutrients and protect against invading pathogens and toxins ${ }^{(7)}$. In addition, the gut microbiota is an essential component of the barrier system and is highly related to epithelial metabolism, proliferation and survival $^{(8)}$. Emerging evidence indicates that these commensal microbes suppress pathogens by competing for access to the epithelial surface and sustain barrier integrity $^{(9,10)}$. Furthermore, some specific bacteria can also release SCFA to modulate multiple physiological processes, especially butyrate, which plays a specific role in the maintenance of gut health ${ }^{(11)}$. However, defective barrier function is associated with a variety of intestinal disorders and diseases ${ }^{(12)}$. Therefore, enhancing the intestinal barrier has become a way for prevention and therapy.

Resistant starch (RS), a type of prebiotic, is defined as a starch that escapes digestion in the small intestine and reaches the large intestine $^{(13)}$. Raw potato starch (RPS) is classified as a type 2 RS (RS2, starch granules). Type 2 resistant starch starches are

Abbreviations: CD, crypt depth; Gcg, proglucagon; $M U C 2$, mucin 2; RS, resistant starch; RPS, raw potato starch; VH, villus height. 
resistant to digestion due to their compact structure that limits the ability of digestive enzymes to access the $\operatorname{starch}^{(14)}$. Currently, there is a growing interest in supplementing RS in human and animal diets due to its beneficial effects on improved gut health ${ }^{(13)}$. Results from previous studies in pigs have suggested that RPS could improve intestinal morphology in the colon and increase the expression of genes responsible for barrier function $^{(10,15)}$. Meanwhile, RS is also a fermentable substrate for caecal and colonic microbes to produce SCFA, such as acetate, propionate and butyrate, and reduce $\mathrm{pH}$ in the caecum ${ }^{(16)}$. Nofrarías et al. $^{(15)}$ found that long-term intake of 25 or $35 \%$ RPS could increase butyrate production and improve colonic mucosal integrity in pigs. In accordance, several studies have shown that mouse or rat diets supplemented with RS could promote gut health, which is likely attributable to an alteration in the microbial community characterised by increased butyrateproducing bacteria, thereby reducing inflammation and maintaining physiologic balance ${ }^{(17)}$. Moreover, Keenan et al. ${ }^{(18)}$ found that rats fed RS had increased gene transcription of proglucagon $(G c g)$ in the caecum. An in vitro study also showed that butyrate could increase $G c g$ gene expression in a dosedependent manner ${ }^{(19)}$. Collectively, these observations suggest an important correlation between dietary RS and intestinal barrier health. Although a considerable amount of research has explored the health-promoting facets of feeding $\mathrm{RS}^{(13)}$, the effects of RS on the gut are extremely complex and much remains to be learned. In the light of this, the aim of the present study was to investigate whether RPS could modulate barrier function and induce shifts in the microbial composition of the caecum in meat ducks.

\section{Methods}

\section{Animals and diets}

All experimental procedures were approved by the Animal Care and Use Committee of Sichuan Agricultural University. A total of 360 Cherry Valley meat-type male ducks of 1-d-old (54.92 (sD 0.23) g; Sichuan Mianying Breeding Duck Co. Ltd) were allocated to three dietary treatments with eight replicates (fifteen birds each) following a completely randomised design: three isoenergetic and isonitrogenous diets with 0 (control), 12 or $24 \%$ purified RPS (AVEBE Ltd). RPS used in the present study contained $54.72 \%$ RS (DM basis). Table 1 shows the diets that were formulated in line with National Research Council guidelines $^{(20)}$. Birds were offered starter (1-14d) and finisher (15-35 d) diets as mash form. All ducks were reared in cages $(2.2 \times 1.2 \times 0.9 \mathrm{~m})$ in a temperature- and humidity-controlled room with feed and water ad libitum throughout the experimental period. Body weight and feed intake per pen were recorded at 1 and $35 \mathrm{~d}$ of age.

\section{Data collection and sampling}

Two birds per pen were sampled at $35 \mathrm{~d}$. One was killed by cervical dislocation, and the length and weight of the full caeca were measured, then the $\mathrm{pH}$ of the caecum was registered
Table 1. Composition and nutrient levels in the basal diets (DM basis)

\begin{tabular}{|c|c|c|c|c|c|c|}
\hline \multirow{2}{*}{$\begin{array}{l}\text { Raw potato starch } \\
\text { (\% of diet weight).. }\end{array}$} & \multicolumn{3}{|c|}{$1-14 d$} & \multicolumn{3}{|c|}{$15-35 d$} \\
\hline & 0 & 12 & 24 & 0 & 12 & 24 \\
\hline \multicolumn{7}{|l|}{ Ingredients } \\
\hline Maize & $59 \cdot 82$ & $44 \cdot 85$ & $29 \cdot 88$ & $69 \cdot 25$ & $53 \cdot 72$ & $38 \cdot 19$ \\
\hline Raw potato starch & - & $12 \cdot 00$ & $24 \cdot 00$ & - & $12 \cdot 00$ & 24.00 \\
\hline Soyabean meal (\%) & $33 \cdot 22$ & 35.91 & $38 \cdot 60$ & 24.93 & $27 \cdot 67$ & 30.41 \\
\hline Soyabean oil & 0.50 & 1.50 & 2.50 & 0.50 & 1.70 & $2 \cdot 90$ \\
\hline Calcium carbonate & $1 \cdot 10$ & 1.04 & 0.98 & 1.07 & 1.01 & 0.95 \\
\hline $\begin{array}{l}\text { Dicalcium } \\
\text { phosphate }\end{array}$ & 1.74 & 1.82 & 1.89 & 1.65 & $1 \cdot 74$ & $1 \cdot 82$ \\
\hline L-Lysine-HCl & 0.11 & 0.07 & 0.02 & 0.04 & - & - \\
\hline DL-Methionine & 0.16 & 0.16 & 0.17 & 0.15 & 0.16 & 0.16 \\
\hline Threonine & 0.02 & 0.01 & 0.01 & - & - & - \\
\hline Tryptophan & - & - & - & 0.01 & 0.01 & 0.01 \\
\hline Bentonite & $2 \cdot 35$ & 1.66 & 0.97 & 1.42 & 1.01 & 0.58 \\
\hline $\mathrm{NaCl}$ & 0.30 & 0.30 & 0.30 & 0.30 & 0.30 & 0.30 \\
\hline Choline chloride & 0.15 & 0.15 & 0.15 & 0.15 & 0.15 & $0 \cdot 15$ \\
\hline Vitamin premix* & 0.03 & 0.03 & 0.03 & 0.03 & 0.03 & 0.03 \\
\hline Mineral premix† & 0.50 & 0.50 & 0.50 & 0.50 & 0.50 & 0.50 \\
\hline $\begin{array}{l}\text { Total } \\
\text { Analysis, calculated } \\
\text { value }\end{array}$ & 100 & 100 & 100 & 100 & 100 & 100 \\
\hline AME (MJ/kg) & 11.72 & $11 \cdot 72$ & $11 \cdot 72$ & $12 \cdot 12$ & $12 \cdot 12$ & $12 \cdot 12$ \\
\hline $\begin{array}{l}\text { CP (\%) } \\
\text { Nutrient analysis (\%) }\end{array}$ & $19 \cdot 50$ & $19 \cdot 50$ & $19 \cdot 50$ & $16 \cdot 50$ & $16 \cdot 50$ & $16 \cdot 50$ \\
\hline Resistant starch & 3.46 & $7 \cdot 31$ & $19 \cdot 70$ & 3.80 & $7 \cdot 37$ & $19 \cdot 46$ \\
\hline
\end{tabular}

AME, apparent metabolism energy; $\mathrm{CP}$, crude protein.

* Provided per kg of diet: vitamin A, $2.06 \mathrm{mg}$; vitamin $D_{3}, 0.04 \mathrm{mg}$; vitamin E, $30.01 \mathrm{mg}$; thiamine, $1 \mathrm{mg}$; riboflavin, $3.9 \mathrm{mg}$; pyridoxine, $3.375 \mathrm{mg}$; vitamin $\mathrm{B}_{12}, 0.01 \mathrm{mg}$; calcium pantothenate, $8.85 \mathrm{mg}$; folate, $0.5 \mathrm{mg}$; biotin, $0.1 \mathrm{mg}$; niacin, $49.25 \mathrm{mg}$. †Provided per kg of diet: $\mathrm{Cu}\left(\mathrm{CuSO}_{4} \cdot 5 \mathrm{H}_{2} \mathrm{O}\right), 8 \mathrm{mg}$; $\mathrm{Fe}\left(\mathrm{FeSO}_{4} \cdot 7 \mathrm{H}_{2} \mathrm{O}\right), 80 \mathrm{mg}$; $\mathrm{Zn}\left(\mathrm{ZnSO}_{4} \cdot 7 \mathrm{H}_{2} \mathrm{O}\right), 90 \mathrm{mg} ; \mathrm{Mn}\left(\mathrm{MnSO}_{4} \cdot \mathrm{H}_{2} \mathrm{O}\right), 70 \mathrm{mg}$; Se $\left(\mathrm{NaSeO}_{3}\right), 0.3 \mathrm{mg}$; iodine $(\mathrm{KI}), 0.4 \mathrm{mg}$.

(pH-STAR, Matthuas, Inc.), and the contents of caeca were removed and weighed. Another bird was anaesthetised, and blood was taken by jugular vein to harvest plasma for endotoxin and cytokines determination. A portion of the caecum was flashfrozen in liquid $\mathrm{N}_{2}$ and stored at $-80^{\circ} \mathrm{C}$ until gene expression analysis, and the remaining caecum were collected for histological determination. Digesta samples were randomly divided into two parts. One portion was stored at $-20^{\circ} \mathrm{C}$ for SCFA determination, and the other was kept in liquid $\mathrm{N}_{2}$ for microbial community analysis.

\section{Resistant starch assay}

RS in RPS and diets was analysed using an RS assay kit (K-RSTAR; Megazyme Ltd).

\section{Plasma endotoxin and cytokine assay}

Plasma concentrations of endotoxins were determined using a commercially available ELISA kit, according to the manufacturer's protocol (Mosak Biotechnology Co., Ltd). This kit was sensitive to $5-\mathrm{pg} / \mathrm{ml}$ endotoxins. Plasma TNF- $\alpha$, IL- $1 \beta$ and IL-10 concentrations were measured using ELISA commercial kits (Mosak Biotechnology Co., Ltd). Cytokines were measured as described by the manufacturer's instructions. All assays were done in duplicate. 


\section{Histology}

Segments of the mid-caecum (about $1.0 \mathrm{~cm}$ ) were removed, flushed gently with ice-cold physiological saline solution and then fixed in $4 \%(\mathrm{w} / \mathrm{v})$ paraformaldehyde, embedded in paraffin, sectioned at $5 \mu \mathrm{m}$ and stained with haematoxylin and eosin by following the standard procedures. The photomicrographs (BA400Digital; Mike Audi Industrial Group Co., Ltd) were obtained to measure villus height (VH), crypt depth (CD) and mucosal thickness using an image analyser (Image Pro-Plus) at $100 \times$ magnification. VH was measured from the tip to the crypt-villus junction, and CD from the crypt-villus junction to the base ${ }^{(21)}$. The mucosal thickness was defined as the distance from the base of muscularis mucosa to the tip of the villus ${ }^{(22)}$.

\section{RNA extraction and real-time $P C R$}

Total RNA was isolated from frozen caecal samples using trizol reagent (Takara), according to the manufacturer's specifications. Primers were designed using Primer 3 (http://bioinfo.ut.ee/ primer3-0.4.0/primer3/) and are shown in online Supplementary Table S1. Real-time quantitative PCR was carried out using the ABI 7500 real-time PCR detection system (Applied Biosystems). All PCR contained $3 \mu \mathrm{l}$ of 10-fold diluted complementary DNA, $1 \mu \mathrm{l}$ each of $5 \mu \mathrm{m}$ forward and reverse primers and $5 \mu \mathrm{l}$ of $1 \times$ SYBR Green Master Mix (Takara). Amplification program was $95^{\circ} \mathrm{C} / 15 \mathrm{~min}$, followed by forty cycles of $95^{\circ} \mathrm{C} / 5 \mathrm{~s}$ and $60^{\circ} \mathrm{C} / 30 \mathrm{~s}$, and a final melting curve analysis. Glyceraldehyde-3-phosphate dehydrogenase and $\beta$-actin were selected as the reference genes, and a normalisation factor was obtained by calculating the geometric mean of the values of the selected reference genes, which was subsequently used to normalise the relative amounts of RNA of interest ${ }^{(23)}$

\section{SCFA analysis}

Approximately $0.5 \mathrm{~g}$ of caecal digesta was diluted with 2-ml ultrapure water, mixed and centrifuged (3000 g, $15 \mathrm{~min}$ ). Supernatant $(1 \mathrm{ml})$ was mixed with $0 \cdot 2-\mathrm{ml}$ ice-cold $25 \%(\mathrm{w} / \mathrm{v})$ metaphosphoric acid solution at $4^{\circ} \mathrm{C}$ for $30 \mathrm{~min}$ and then centrifuged. Concentrations of acetate, propionate and butyrate were measured by GC system (Varian CP-3800).

\section{DNA extraction and sequencing of 165 rRNA}

Total genomic DNA from samples was extracted using QIAamp PowerFecal DNA kit (Qiagen), according to the manufacturer's instructions. DNA concentration and quality were checked using a NanoDrop Spectrophotometer. DNA was diluted to $10 \mathrm{ng} / \mu \mathrm{l}$ using sterile ultrapure water and stored at $80^{\circ} \mathrm{C}$ for downstream use. The V4 region of the $16 \mathrm{~S}$ rRNA genes (Primer-515F: 5'-GTGCCAGCMGCCGCGGTAA-3'; -806R: 5'-GGACTACHVGGGTWTCTAAT-3') was amplified using the specific primer with 12 nucleotide (nt) unique barcode (25). The PCR mixture $(25 \mu \mathrm{l})$ contained $1 \times$ PCR buffer, $1.5-\mathrm{mm}$ $\mathrm{MgCl}_{2}$, each deoxynucleoside triphosphate at $0.4 \mu \mathrm{m}$, each primer at $1.0 \mu \mathrm{m}, 0.5 \mathrm{U}$ of KOD-Plus-Neo (TOYOBO) and 10-ng template DNA. The PCR amplification program consisted of initial denaturation at $94^{\circ} \mathrm{C}$ for $1 \mathrm{~min}$, followed by thirty cycles (denaturation at $94^{\circ} \mathrm{C}$ for $20 \mathrm{~s}$, annealing at $54^{\circ} \mathrm{C}$ for $30 \mathrm{~s}$ and elongation at $72^{\circ} \mathrm{C}$ for $30 \mathrm{~s}$ ) and a final extension at $72^{\circ} \mathrm{C}$ for $5 \mathrm{~min}$. Three replicates of PCR for each sample were pooled. PCR products mixed with one-sixth volume of $6 \times$ loading buffer and then loaded on $2 \%$ agarose gels and purified using QIAquick Gel Extraction kit (Qiagen) following the manufacturer's recommendations and quantified using Qubit@ 2.0 Fluorometer (Thermo Scientific) and pooled with an equal molar amount. The library was applied to paired-end sequencing $(2 \times 250 \mathrm{bp})$ with the Illumina Hiseq apparatus at Rhonin Biosciences Co., Ltd.

\section{Sequence processing}

The sequences were analysed according to Usearch (http:// drive5.com/uparse/) and QIIME pipeline ${ }^{(24)}$. Reads from the original DNA fragments were merged using FLASH ${ }^{(25)}$, low-quality reads were filtered $(q<30)$ and potential chimeric sequences were removed using the Uchime algorithm ${ }^{(26)}$. After finding duplicate sequences, all the singletons were discarded due to their possible bad amplicons (http://www.drive5.com/ usearch/manual/singletons.html), which may lead to an overestimation of diversity. Sequences were clustered into operational taxonomic units at $97 \%$ identity threshold based on the UPARSE algorithm in Usearch ${ }^{(27)}$. Taxonomy were assigned using the SILVA database (Release_123) ${ }^{(28)}$ and uclust classifier in QIIME with default parameters. Representative sequences were aligned using PyNAST ${ }^{(29)}$. A phylogenetic tree was constructed using the generalised time-reversible model in FastTree $^{(30)}$. The relative abundance of the taxon at each taxonomic level was produced based on the operational taxonomic unit abundance and taxonomic annotation. $\alpha$-Diversity and $\beta$-diversity metrics were calculated using R Vegan package ${ }^{(31)}$. Weighted and unweighted Unifrac distances were calculated in R GUniFrac package to determine the degree of similarity between samples and used to perform principal coordinate analysis. Random forest analysis was applied to obtain the important indicator taxa using $\mathrm{R}$ random Forest package with 1000 trees and other default parameters.

\section{Statistical analysis}

Data were analysed using one-way ANOVA of the mixed model procedure of SAS 9.4 software (SAS Institute, Inc.). The independent variable was the RPS treatment. When significant, post boc comparisons of treatment means were made using Tukey's test. Statistical significance was detected at $P<0 \cdot 05$. Figures were generated by Prism 7.0 (GraphPad Software, Inc.). All data were expressed as means and standard errors.

\section{Results}

\section{Caecal parameters and morphology}

Neither final body weight nor daily feed intake was affected by the dietary RPS during the 35-d study period (online Supplementary Table S2). The length and weight of caecum increased with dietary RPS supplementation and significantly increased in the $24 \%$ RPS diet when compared with the control diet $(P<0.05)$. The $\mathrm{pH}$ of caecal contents was notably lower in ducks fed the $12 \%$ RPS diet compared with the control diet. The caecal digesta 
Table 2. Effect of dietary raw potato starch (RPS) concentration on caecal parameters and morphology in meat ducks (Mean values with their standard errors $(n 8)$ )

\begin{tabular}{|c|c|c|c|c|c|c|c|}
\hline \multirow[b]{3}{*}{ Item } & \multicolumn{6}{|c|}{ Raw potato starch (\% of diet weight) } & \multirow[b]{3}{*}{$P$} \\
\hline & \multicolumn{2}{|c|}{0} & \multicolumn{2}{|c|}{12} & \multicolumn{2}{|c|}{24} & \\
\hline & Mean & SE & Mean & SE & Mean & SE & \\
\hline Length (cm) & $29 \cdot 13^{b}$ & 0.92 & $32 \cdot 4^{a, b}$ & 1.09 & $33 \cdot 86^{\mathrm{a}}$ & 1.08 & 0.022 \\
\hline Weight $(\mathrm{g})$ & $3.00^{\mathrm{b}}$ & 0.12 & $3.42^{a, b}$ & 0.12 & $3.63^{a}$ & 0.12 & 0.007 \\
\hline Content (g) & 1.44 & 0.17 & 1.99 & 0.34 & $1 \cdot 70$ & 0.10 & 0.313 \\
\hline $\mathrm{pH}$ & $6 \cdot 41^{\mathrm{a}}$ & 0.07 & $5 \cdot 80^{\mathrm{b}}$ & $0 \cdot 19$ & $6 \cdot 10^{a, b}$ & $0 \cdot 10$ & 0.013 \\
\hline Villus height $(\mu \mathrm{m})$ & $271.35^{\mathrm{b}}$ & $8 \cdot 14$ & $339.66^{a}$ & $18 \cdot 27$ & $330 \cdot 44^{\mathrm{a}}$ & $13 \cdot 61$ & 0.004 \\
\hline Crypt depth $(\mu \mathrm{m})$ & $109 \cdot 71^{a}$ & 5.00 & $92 \cdot 29^{a, b}$ & 6.36 & $87.59^{b}$ & 5.42 & 0.022 \\
\hline $\mathrm{VH}: \mathrm{CD}$ & $2.55^{\mathrm{b}}$ & 0.13 & $4.02^{\mathrm{a}}$ & 0.21 & $4.09^{a}$ & 0.24 & $<0.001$ \\
\hline Mucosal thickness $(\mu \mathrm{m})$ & $402 \cdot 18^{b}$ & $12 \cdot 33$ & $475 \cdot 1^{\mathrm{a}}$ & 23.07 & $451 \cdot 01^{\mathrm{a}, \mathrm{b}}$ & $15 \cdot 28$ & 0.048 \\
\hline
\end{tabular}

$\mathrm{VH}: \mathrm{CD}$, villus height:crypt depth ratio.

a,b Mean values in a row with unlike superscript letters were significantly different $(P<0.05$; one-way ANOVA and Tukey's multiple range test).

Table 3. Effect of dietary raw potato starch on the diversity of caecal digesta microbiota in ducks

(Mean values with their standard errors $(n 8)$ )

\begin{tabular}{|c|c|c|c|c|c|c|c|}
\hline \multirow[b]{3}{*}{ Item } & \multicolumn{6}{|c|}{ Raw potato starch (\% of diet weight) } & \multirow[b]{3}{*}{$P$} \\
\hline & \multicolumn{2}{|l|}{0} & \multicolumn{2}{|c|}{12} & \multicolumn{2}{|l|}{24} & \\
\hline & Mean & SE & Mean & SE & Mean & SE & \\
\hline Chao 1 & $1288 \cdot 32^{a, b}$ & 112.90 & $1381.98^{a}$ & 60.72 & $1011 \cdot 16^{b}$ & 60.29 & 0.012 \\
\hline Ace & $1336 \cdot 19^{a, b}$ & $123 \cdot 36$ & $1426 \cdot 27^{\mathrm{a}}$ & 71.52 & $1039 \cdot 61^{b}$ & 66.98 & 0.017 \\
\hline Shannon & $4.12^{b}$ & 0.17 & $4 \cdot 61^{a}$ & 0.08 & $3.89^{b}$ & 0.05 & 0.001 \\
\hline Simpson & $0.90^{\mathrm{b}}$ & 0.02 & $0.96^{a}$ & 0.01 & $0.91^{a, b}$ & 0.01 & 0.021 \\
\hline
\end{tabular}

a,b Mean values in a row with unlike superscript letters were significantly differen $(P<0.05$; one-way ANOVA and Tukey's multiple range test).

contents did not differ among groups (Table 2). Ducks fed the RPS-containing diets had increased $\mathrm{VH}$ and ratio of VH:CD and decreased $\mathrm{CD}$ in the caecum compared with those fed the $0 \%$ RPS diet $(P<0.05)$. The mucosal thickness was significantly increased by the $12 \%$ RPS diet in the present study $(P<0.05)$.

\section{Diet-related differences in microbial community}

Illumina Hiseq high-throughput sequencing was performed to compare the microbial communities in the caecum among the three dietary groups. A total of 665392 sequencing reads were obtained from the caecal digesta samples, and 5139 operational taxonomic units were identified in the caecum. There were no differences in richness estimators (Chao 1 and Ace) or diversity indices (Simpson and Shannon) of the caecal microbiota between the RPS diets and the control group (Table 3). Microbial distribution was then examined by principal coordinate analysis clustering using weighted Unifrac distance ( $\beta$-diversity), which indicated separate clustering of caecal bacterial communities by diets (Fig. 2(A)). The overall commensal structures of the 0, 12 and $24 \%$ groups were different because of the influence of the diets. Additionally, pairwise variation among samples was evaluated based on their treatments by weighted Unifrac distances (Fig. 2(B)). Pairwise distance between the 12 and $24 \%$ RPS groups (12_24) was significantly greater than intra-group (0_0,12_12, and 24_24) and inter-group (0_12 and 0_24) $\beta$-diversity, 0 and $24 \%$ RPS groups (0_24) was significantly higher than intra-group (0_0 and 12_12), and the 0 and $12 \%$ RPS groups (0_12) was significantly greater than intragroup (12_12). This difference in pairwise difference indicates a distinct role of RPS in altering the microbial structures.

The relative taxa abundance of bacteria was analysed from the phylum to genus level (Fig. 1 and online Supplementary Fig. S1). Bacteroidetes and Firmicutes were the two most dominant phyla in the duck's caecal digesta (Fig. 1(A)). After supplementation with $12 \%$ RPS in the diet, ducks had a distinctively higher abundance of Firmicutes compared with that of the control and $24 \%$ RPS groups $(P<0 \cdot 05)$. Additionally, Proteobacteria $(P<0.05)$ was significantly increased in the $24 \%$ RPS group (Fig. 2(C)).

According to random forest analysis (Fig. 2(D)), the relative abundance of several genera within the phylum Firmicutes, including Faecalibacterium, Ruminococcaceae UCG-014, Subdoligranulum, Erysipelatoclostridium, Ruminococcaceae UCG-010 and Ruminiclostridium 5 was significantly higher in caecal digesta from birds fed the $12 \%$ RPS compared with those fed the $0 \%$ RPS diet. Furthermore, genera such as Lactobacillus and Bifidobacterium as well as Enterobacter were enriched in the digesta of birds fed the $24 \%$ RPS diet, while Oscilospira and Subdoligranulum were relatively deficient compared with that of $0 \%$ groups. Both 12 and $24 \%$ RPS were lower in the Rikenellaceae RC9 gut group and Alloprevotella.

\section{Caecal digesta SCFA profiles}

Acetate, propionate and butyrate contents were greater in ducks that were fed the $12 \%$ RPS diet compared with the $0 \%$ RPS diet $(P<0 \cdot 05)$, and the SCFA contents of caecal digesta were inconspicuous when the RPS dose was added to $24 \%$, though the value in the $24 \%$ RPS diet was still higher than that in the control diet (Fig. 3).

\section{Gene expression of barrier-related genes in the caecum}

The caecal mucosal mucin (MUC)2, Gcg and tight junctionrelated gene mRNA expression levels of ducks are presented in Fig. 4. Supplementation with RPS-containing diets significantly up-regulated the expression of MUC2 and Gcg mRNA (Fig. 4(A) and (B)). When compared with the $0 \%$ RPS diet, the 24 and $12 \%$ RPS diets induced a significantly higher zonula occludens- 1 and claudin 1 mRNA level, respectively (Fig. 4(C) and (E)). Dietary 
(A)

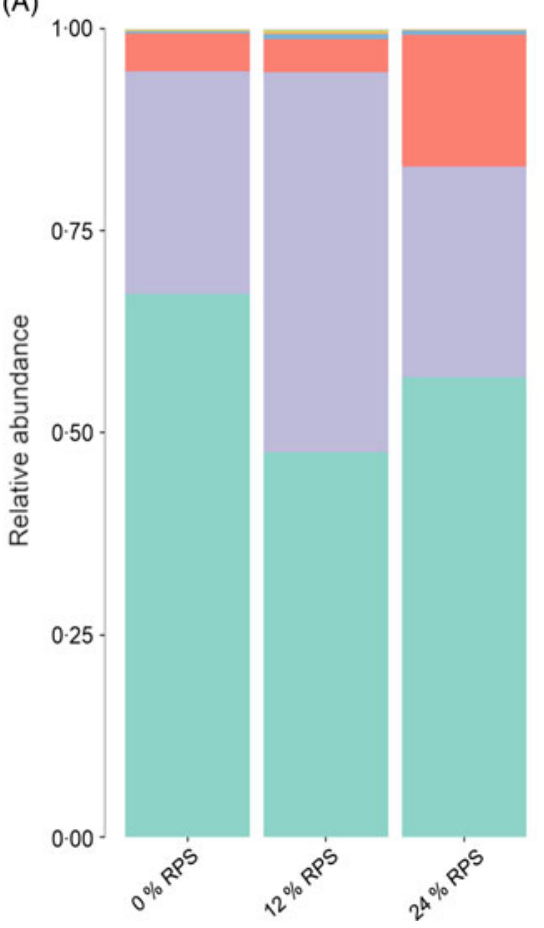

(B)

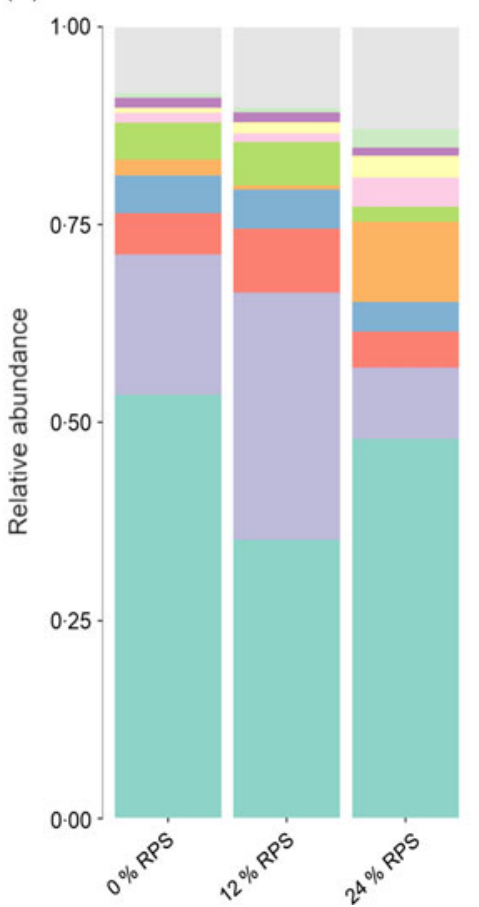

(C)

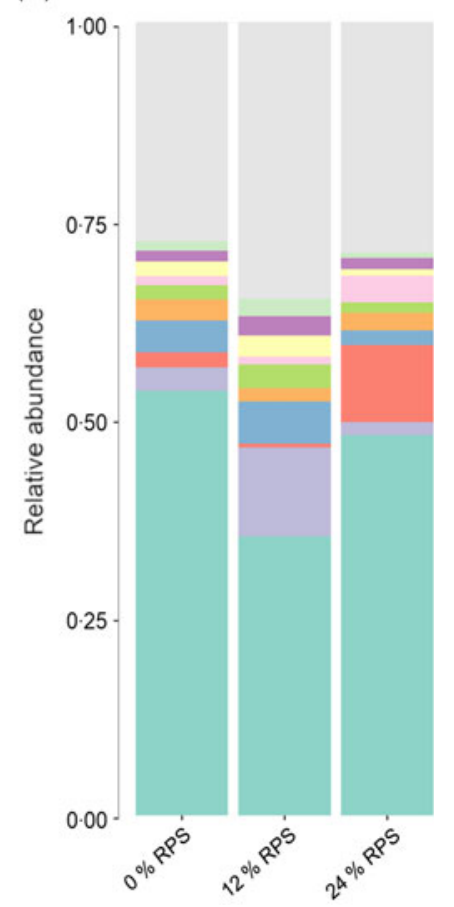

Fig. 1. Summary of bacterial taxa in duck caecal digesta observed by the concentration of raw potato starch (RPS) in the diet. The relative abundances of bacterial $97 \%$ operational taxonomic units (OTU) are shown for duck caecal digesta samples grouped by RPS concentration. (A) Depiction of phylum-level classifications for observed OTU. Phylum: , others; , Acidobacteria; _, Chlorobi; , Fusobacteria; , Verrucomicrobia; , Deferribacteres; , Tenericutes; , Actinobacteria; , Proteobacteria; , Firmicutes; , Bacteroidetes. (B) Depiction of family-level classifications for observed OTU. Family: , others; , Enterococcaceae; , Bacteroidales S24-7 group;

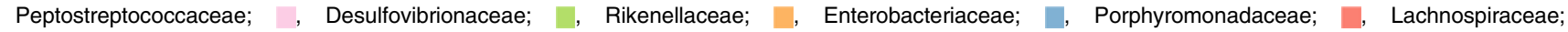
[, Ruminococcaceae; , Bacteroidaceae. (C) Depiction of genus-level classifications for observed OTU for samples grouped by dietary RPS concentration. Genus: , others; , Subdoligranulum; , Lachnoclostridium; , [Eubacterium] coprostanoligenes group; , Desulfovibrio; , Barnesiella; , Parabacteroides; , Alistipes; [, Escherichia-Shigella; [, Faecalibacterium; [, Bacteroides.

RPS administration did not alter the transcription level of occludin among groups (Fig. 4(D)).

\section{Plasma endotoxin and cytokine contents}

The concentrations of plasma endotoxin, TNF- $\alpha$, IL- $1 \beta$ and IL-10 are shown in Fig. 5. Ducks fed either the 12 or the $24 \%$ RPS diet showed reduced plasma levels of endotoxin, TNF- $\alpha$ and IL- $1 \beta$ when compared with the $0 \%$ RPS diet, and no significant difference was observed for endotoxin and TNF- $\alpha$, IL- $1 \beta$ between the RPS-containing diets (Fig. 5(A)-(C)). IL-10, however, was unaffected by dietary RPS contents (Fig. 5(D)).

\section{Discussion}

The normal development of the hind gut is the foundation for maintaining barrier function and fermentability ${ }^{(32)}$. Recent research showed that RPS could increase the weight of the large intestine and the mucosa thicknesses of the caecum in pigs $^{(33)}$. In our study, an increase in VH, VH:CD and mucosal thickness was observed following RPS supplements and indicated an enhanced absorptive capacity and intestinal health ${ }^{(6)}$. The intestinal $\mathrm{pH}$ is largely affected by the amount of dietary fermentable carbohydrates entering the large intestine and has been used as an indicator for intestinal health ${ }^{(34)}$. Our results showed that feeding the RPS-containing diets led to a lower $\mathrm{pH}$ in the caecal digesta. These results correspond with those of meta-regression analysis by Metzler-Zebeli et al ${ }^{(35)}$ which found that, with a minimum amount of 10-15\% RS in the diet, type 2 resistant starch effectively decreased the $\mathrm{pH}$ in the hind gut of pigs. These results suggest that dietary RPS supplementation seems to have positive effects on the caecal development of ducks.

The health-promoting effects of RS may be mediated through improvement in microbiota composition. The microbiota within the hind gut plays a distinct role in defence against pathogens and maintenance of intestinal health ${ }^{(36)}$. In the present study, diets did not affect the predominant phyla in the caecum, which is consistent with previous studies of ducks and chickens that Bacteroidetes and Firmcutes were the two dominant phyla in the caecum, with Bacteroidetes at a higher proportion ${ }^{(37,38)}$. However, several studies of prebiotics have shown expansion of Firmicutes ${ }^{(39)}$ or higher Firmicutes:Bacteroidetes ratio ${ }^{(40)}$. It was demonstrated that the abundance of Firmicutes had a strong negative correlation with pathogenic bacterial populations in the intestine ${ }^{(41)}$. Concomitant with these observations, RPS induced an increase in Firmicutes proportions mainly from a bloom in the relative abundance of families Lachnospiraceae and Ruminococcaceae. Both of these families are linked with gut health for their butyrate-producing properties ${ }^{(42)}$. It is well established that changes in gut microbiota composition is highly associated with nutrient absorption and SCFA production. For example, members of Ruminococcaceae harbour a unique ability to increase the utilisation of $\operatorname{starch}^{(43)}$; Subdoligranulum, 
(A) PCOA based on weighted unifrac distance

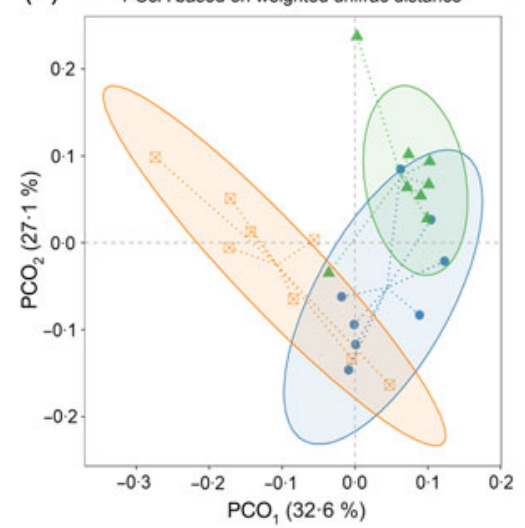

(B)

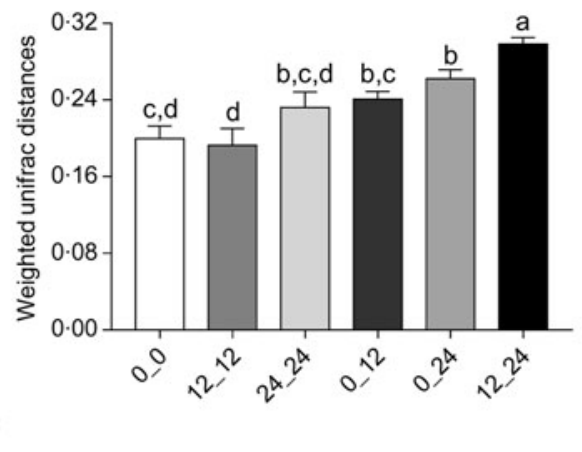

(C)

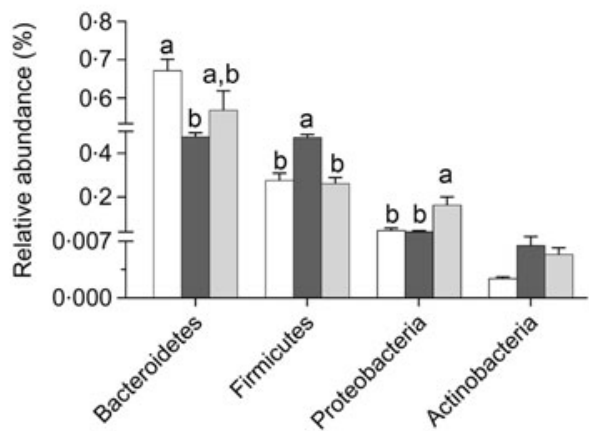

(D)

$$
\text { g_Faecalibacterium - }
$$

g_Rikenellaceae RC9 gut group -

g_Ruminococcaceae UCG-014 -

g_Ralstonia -

g_Subdoligranulum -

g_Oscillospira -

g__[Eubacterium] nodatum group -

g_Lactobacillus -

g_Alloprevotella -

g_Enterobacter -

g_Streptococcus -

g_Erysipelatoclostridium -

g_Anaerofilum -

g_Bifidobacterium -

g_Ruminococcaceae UCG-002 -

g_Ruminococcaceae UCG-010-

g_Pseudomonas -

g_Ruminiclostridium 5-

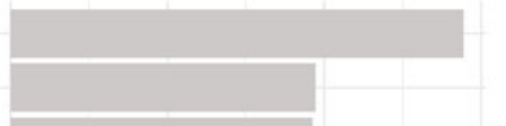

$\sqrt{10}$
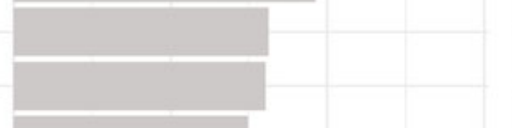

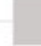

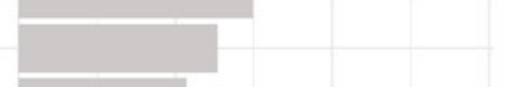

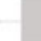
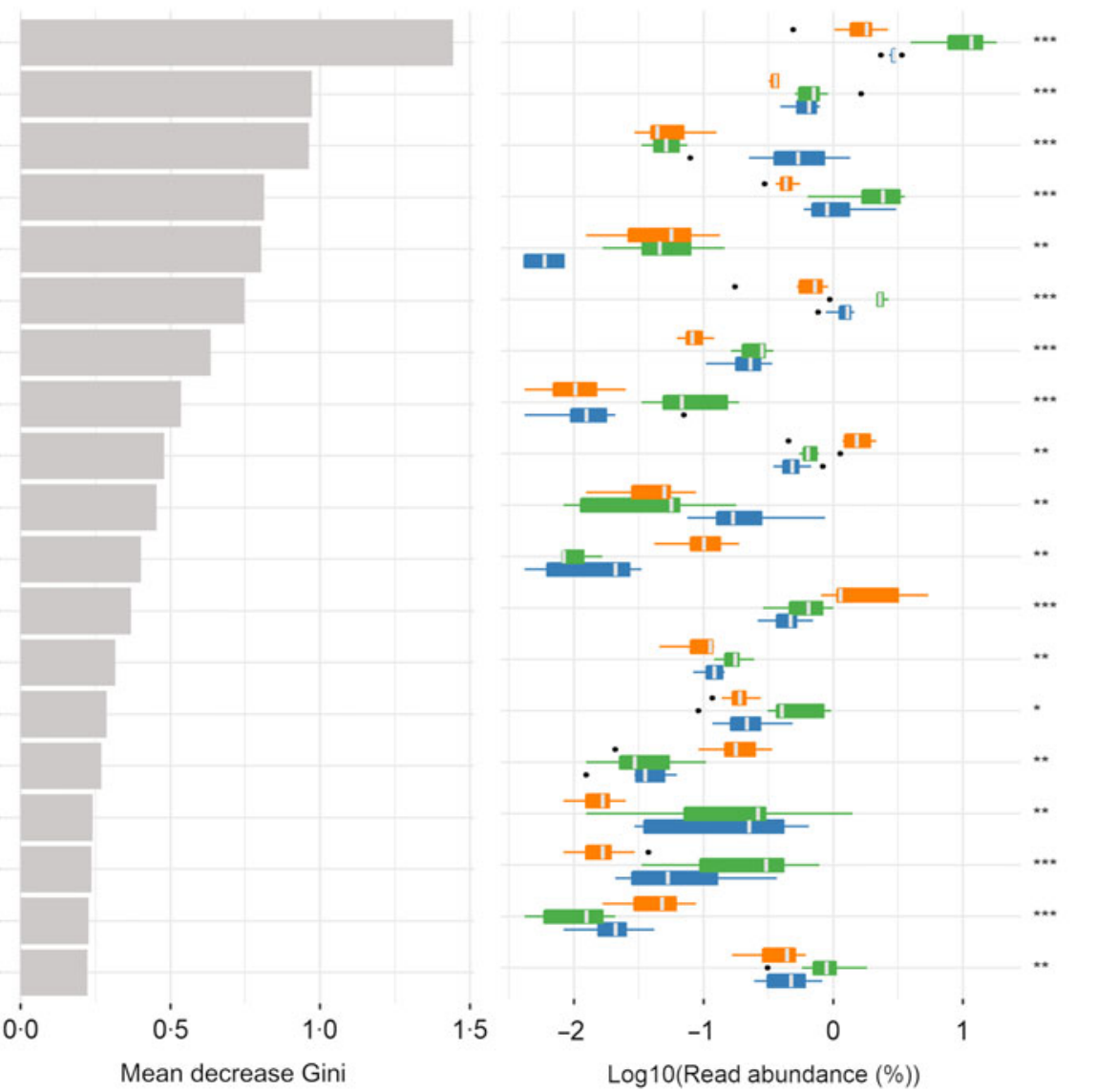

Fig. 2. Alteration of the microbial community of caecal digesta of ducks fed 0,12 , or $24 \%$ raw potato starch (RPS). (A) Principal coordinate analysis (PCoA) among the three groups based on weighted UniFrac distances. Each point represented a sample. Group: $\bullet, 0 \%$ RPS, $\triangle, 12 \%$ RPS, 圆, $24 \%$ RPS. (B) Comparisons of pairwise weighted Unifrac distances. ANOVA was conducted for multiple comparisons with the least significant difference method for correction. 0: $0 \%$ RPS; $12: 12 \%$ RPS; 24 : $24 \%$ RPS. 0_0, 12_12 and 24_24 indicate intra-group distances; 0_12, 0_24 and 12_24 indicate inter-group distances. Data are means, with their standard errors represented by vertical bars. (C) Relative abundance of taxa at the phylum level in $0 \%$ RPS (control), $12 \%$ RPS and $24 \%$ RPS. Significance was determined by one-way ANOVA. Data are means, with their standard errors represented by vertical bars. $=, 0 \%$ RPS, $\square, 12 \%$ RPS, $\square, 24 \%$ RPS. (D) Gini index in random forest classification. The left panel represents the mean decreasing Gini index of each taxon in random forest analysis, and the right panel represents the relative abundance of bacteria in each group. Relative abundance was log10 transformed. Significance was determined by the Kruskal-Wallis rank sum test. * $P<0.05$, ${ }^{\star *} P<0.01$ and ${ }^{* * *}$ $P<0.001$. Group: 市, $24 \%$ RPS; 市, $12 \%$ RPS; 0 \% RPS.

Faecalibacterium and Erysipelatoclostridium are butyrate producers ${ }^{(44,45)}$, belonging to Firmicutes, with a higher abundance in the $12 \%$ RPS group. Sun $e t$ al. ${ }^{(46)}$ also reported that pigs fed an RPS diet increased the abundances of butyrate-producing bacteria. Research has also shown that dietary RPS can elevate butyrate concentration of the hind gut in pigs ${ }^{(10,33)}$. In the present study, it was evident that changes in butyrate content occurred after feeding the RPS-containing diets was concurrent with an increase in the abundance of butyrate-producing bacteria, as shown in our study. In addition, the abundance of Lactobacillus 


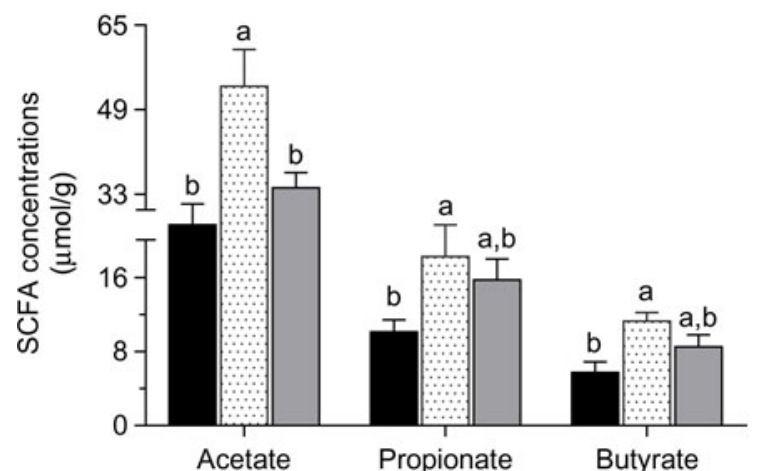

Fig. 3. SCFA concentrations of acetate, propionate and butyrate in ducks fed differing concentrations of raw potato starch (RPS). Values are means, with their standard errors represented by vertical bars. ${ }^{a, b}$ Mean values with unlike letters were significantly different $(P<0.05$; one-way ANOVA and Tukey's multiple range test). $\square, 0 \%$ RPS; $\ldots . . ., 12 \%$ RPS; $\square, 24 \%$ RPS.

and Bifidobacterium, both well-known lactate-producing bacteria, was significantly enriched in ducks fed the $24 \%$ RPS diet. Our observations are consistent with previous high throughput sequencing-based studies, with an increase in Bifidobacterium proportions following RS supplementation ${ }^{(47,48)}$. It was suggested that the major end products of Bifidobacterium fermentation, acetate and lactate, can be co-metabolised by other bacterium to form butyrate ${ }^{(49)}$. The elevation in the abundance of butyrate-producing bacteria is further supported by increased butyrate and acetate concentrations in the caeca of ducks fed the $12 \%$ RPS diet. However, it seems that the relative abundance of Lactobacillus and Bifidobacterium was too low to influence SCFA production, which resulted in the expected increase in acetate and butyrate concentrations as $12 \%$ RPS not being reached after feeding $24 \%$ RPS. These differences in SCFA stimulation may be partially explained by the lowered Oscillospira and Alloprevotella proportion in the $24 \%$ RPS group. Since Oscillospira are butyrate producers, and Alloprevotella, a member of the Prevotellaceae family, also has the ability to produce SCFA in digestion of carbohydrates $^{(50,51)}$. Another important observation in the present study was the increased abundance of Enterobacteriaceae in the caecum, due to the fact that SCFA could directly reduce the growth of Enterobacteriaceae by lowering the $\mathrm{pH}$ in the gut ${ }^{(51)}$. Nonetheless, given both microbiota composition and SCFA concentration observation, we believe that feeding RPS diets may have a favourable effect on barrier development in the caecum.

Butyrate is an important metabolite in the hind gut, arising from bacterial fermentation. Apart from providing energy for intestinal epithelial cell growth, butyrate also has been reported to improve gut barrier function ${ }^{(52)}$. It has been reported that MUC2 synthesis is markedly elevated after butyrate stimulation ${ }^{(53)}$. MUC2 is the main mucus barrier component that covers the intestinal mucosa to maintain an effective mucus layer ${ }^{(54)}$. Trachsel et al. ${ }^{(10)}$ reported that an RPS diet could increase butyrate concentration and the expression of $M U C 2$ in the caecum. Similar to previous work, we observed a higher $M U C 2$ gene expression in RPS-containing diet groups, probably attributed to the elevation of butyrate production. Furthermore, tight junctions are primarily responsible for intestinal permeability ${ }^{(55)}$. In the present study with meat ducks, feeding of 12 and $24 \%$ RPS significantly increased the mRNA expression of claudin 1 and zonula occludens-1, respectively, which are the two main regulators of tight junction permeability. A promising candidate for promoting claudin 1 and zonula occludens- 1 mRNA up-regulation was also the increased butyrate content when birds were fed RPS diets. Studies have shown that treatment with butyrate stimulated the expression of zonula occludens- 1 and claudin $1^{(52,56)}$. Intriguingly, there was a significantly higher mRNA level of $G c g$ in the caecum from the RPS-fed groups in the present study. As known, Gcg is a precursor for glucagon-like peptide (GLP)-1 and GLP-2 that are secreted from enteroendocrine L cells, which have the ability to improve intestinal barrier function ${ }^{(57)}$. It has been reported that butyrate stimulated the GLP-2 secretion through up-regulation of $G c g$ mRNA expression ${ }^{(58)}$. GLP-2 stimulation has been shown to have a beneficial effect on intestinal morphology, VH and crypt cell proliferation in a study of broilers ${ }^{59)}$. At this point, this evidence provides an indirect but strong hint at the improvement of caecal morphology induced by RPS supplementation.

As a primary barrier between the external environment and gut internal milieu, the mucosa defends against numerous pathogen and noxious substances. A thinner and more permeable mucosal layer may increase endotoxin production and passage, thus causing defective barrier function ${ }^{(7,60)}$. Disturbances in the intestinal barrier often trigger excessive release of pro-inflammatory cytokines, leading to an inflammatory response ${ }^{(12)}$. TNF- $\alpha$, one of the major pro-inflammatory cytokines, promotes claudin 1 removal from tight junctions, accelerates occludin degradation and enhances myosin light-chain kinase phosphorylation, thus augmenting paracellular permeability ${ }^{(61)}$. IL- $1 \beta$, as a proinflammatory cytokine, also plays a crucial role in inflammatory reactions ${ }^{(62)}$. The present data showed that ducks fed diets with RPS had reduced plasma endotoxin, TNF- $\alpha$ and IL- $1 \beta$ concentration in meat ducks when compared with ducks fed the $0 \%$ RPS diet. Our observations are consistent with the previous studies that showed that RS in diets decreased serum TNF- $\alpha$ levels in mice ${ }^{(63)}$. Knowledge of the mechanism by which RPS regulates inflammatory cytokines is still extremely limited. Our results demonstrated that enhanced caecal barrier function and elevated SCFA production may be achieved by feeding RPS, at least, play an impotent role in alleviating inflammation. A previous report reviewed altered intestinal permeability and bacterial translocation may result in intestinal infections and inflammation $^{(7)}$. Moreover, Zhou et al. ${ }^{(64)}$ reported that butyrate can be an anti-inflammatory agent in the chicken. Collectively, our observations strongly provide an important link between the RPS-mediated changes in inflammatory responses to improve caecal barrier function of ducks.

The present study raises a new question about the dose of RPS used in meat duck for promoting caecal barrier function. The evidence of this work clearly demonstrates that diet supplemented with $12 \%$ RPS partially improved caecal barrier function of meat ducks compared with $0 \%$ RPS, but the positive role of RPS would be inconspicuous when the dose of RPS was added to $24 \%$, and it was not even an obvious difference between the 24 and $0 \%$ RPS diets. For example, no statistical increases with the dietary increasing RPS in terms of SCFA contents. 
(A)

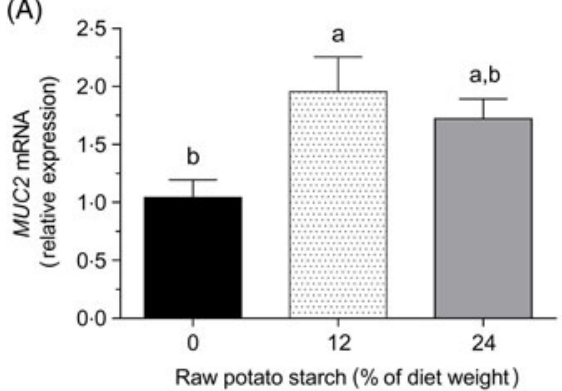

(C)

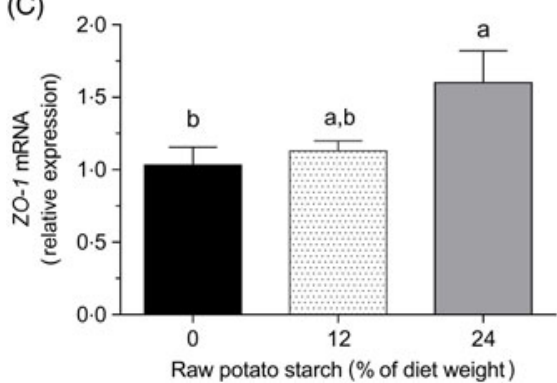

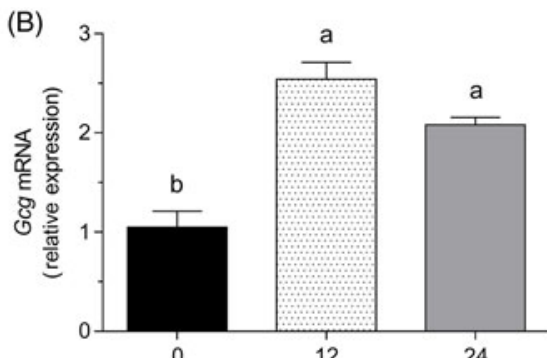

Raw potato starch (\% of diet weight)

(D)

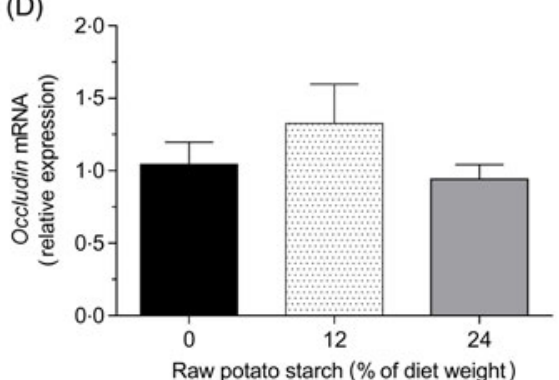

(E)

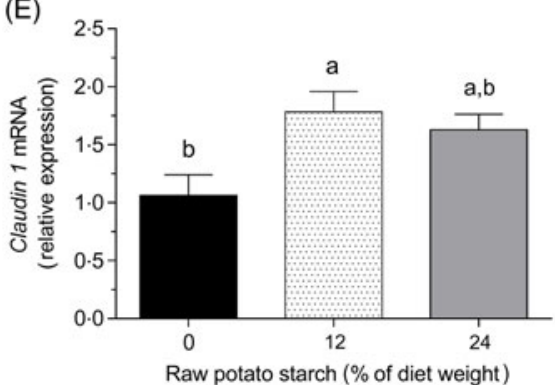

Fig. 4. Expression of mRNA levels involved in caecal barrier function, including (A) mucin (MUC)-2, (B) proglucagon (Gcg), (C) zonula occludens (ZO)-1, (D) occludin and $(E)$ claudin 1 of ducks fed differing concentrations of raw potato starch diets. Values are means, with their standard errors represented by vertical bars. ${ }^{a, b}$ Mean values with unlike letters were significantly different $(P<0.05$; one-way ANOVA and Tukey's multiple range test).

(A)

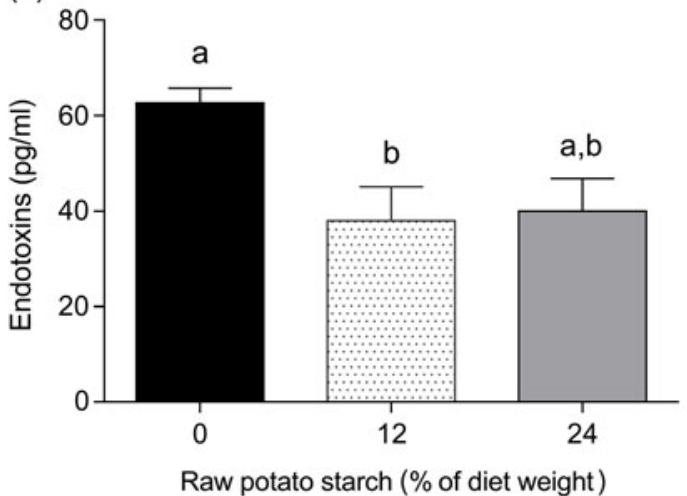

(C)

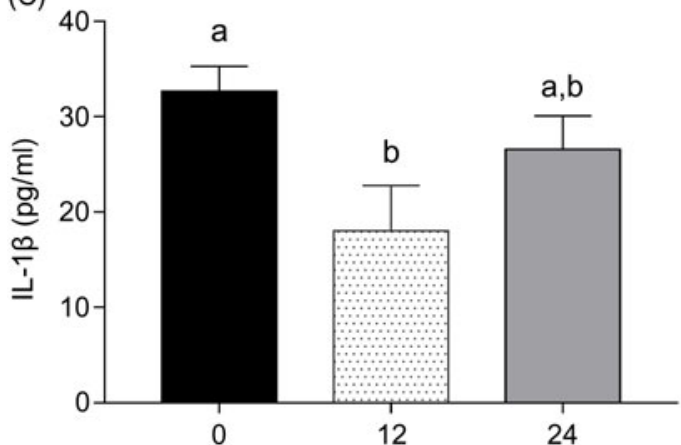

Raw potato starch (\% of diet weight)
(B)

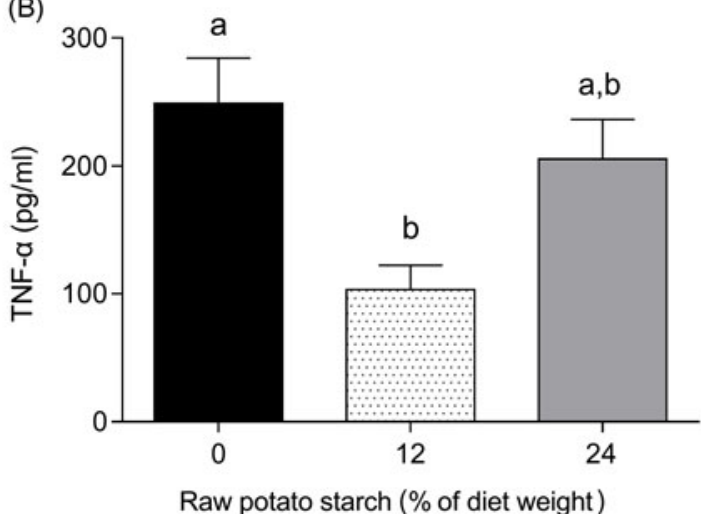

(D)

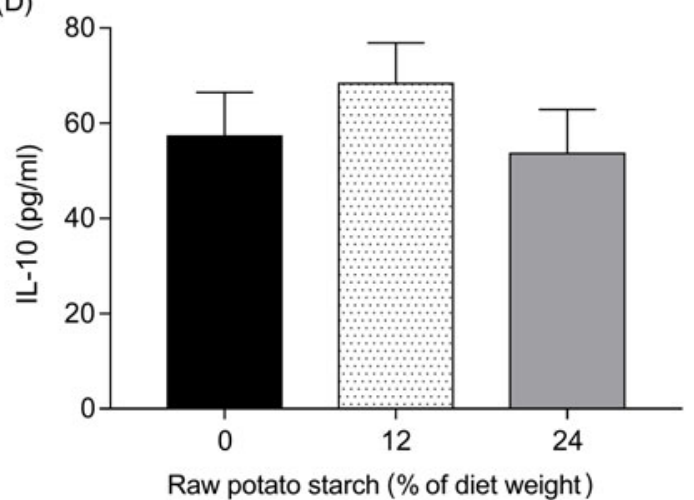

Fig. 5. Plasma (A) endotoxin, (B) TNF- $\alpha$, (C) IL-1 $\beta$ and (D) IL-10 concentration of ducks consuming diets with different concentrations of raw potato starch. Values are means, with their standard errors represented by vertical bars. ${ }^{a, b}$ Mean values with unlike letters were significantly different $(P<0.05$; one-way ANOVA and Tukey's multiple range test). 
However, recent findings in mammals suggested that the contents of SCFA were also significantly increased when supplemented with more than $20 \% \mathrm{RS}^{(33,65)}$. It indicated that there might be an extremely complex mechanism underlying the relationship between the RPS and intestinal barrier function in meat ducks. Either specific structure of the digestive tract of poultry or difference in the sensitivity of ducks to RS could be possible factors. Thus, further studies are needed to validate this possibility.

In conclusion, we demonstrated that $12 \%$ RPS supplement in the diet could enhance barrier function in the caecum of 35-d-old ducks. Significant biomarkers including improved caecal morphological parameters, altered microbial composition and increased expression of barrier markers as well as decreased plasma inflammatory markers were identified and modulated by supplementing dietary RPS. These results also strongly indicate that alterations in caecal microbial composition may result in an increase in butyrate concentration and subsequently impart positive effects on the intestinal barrier. Further studies are needed to elucidate the host response to dietary RPS supplementation in ducks undergoing intestinal challenges to further understand microbiota alterations and the effects of RPS on intestinal barrier function.

\section{Acknowledgements}

The authors thank Gregory Fraley for the revision of English. The raw pyrosequencing reads were submitted to Sequencing Read Archive (SRA) data base under the accession id: SRR7370079.

The research was supported by the National Natural Science Foundation of China (31772622), China Agriculture Research System (CARS-42-10), National Key R \& D Program of China (2017YFD0502004), the 111 Project and Sichuan Agricultural University 211 Foundation of China.

S. Q., Q. Z. and K. Z. designed the experiment. S. Q., Z. S., Y. X., X. D. and H. P. conducted the experiment. S. Q., J. W., S. B. and Y. L. collected and analysed data. S. Q., T. J. A. and Q. Z. wrote the manuscript.

The authors declare that there are no conflicts of interest.

\section{Supplementary material}

To view supplementary material for this article, please visit https://doi.org/10.1017/S0007114519002319

\section{References}

1. Qin S, Tian G, Zhang KY, et al. (2017) Influence of dietary rapeseed meal levels on growth performance, organ health and standardized ileal amino acid digestibility in meat ducks from 15 to 35 days of age. J Anim Physiol Anim Nutr 101, 1297-1306.

2. Park SO \& Kim WK (2017) Effects of betaine on biological functions in meat-type ducks exposed to heat stress. Poult Sci $\mathbf{9 6}$, 1212-1218.

3. Bi Y, Nan XM, Zheng SS, et al. (2018) Effects of dietary threonine and immune stress on growth performance, carcass trait, serum immune parameters, and intestinal muc2 and NF-kb gene expression in Pekin ducks from hatch to 21 days. Poult Sci $97,177-187$.

4. He J, Zhang K, Chen D, et al. (2013) Effects of maize naturally contaminated with aflatoxin $\mathrm{B} 1$ on growth performance, blood profiles and hepatic histopathology in ducks. Livest Sci $\mathbf{1 5 2}$, 192-199.

5. Bai WQ, Zhang KY, Ding X, et al. (2019) High dietary energy content increases inflammatory markers after lipopolysaccharide challenge in meat ducks. Poult Sci 98, 164-171.

6. Ducatelle R, Goossens E, De Meyer F, et al. (2018) Biomarkers for monitoring intestinal health in poultry: present status and future perspectives. Vet Res 49, 43.

7. De Medina FS, Romerocalvo I, Mascaraque C, et al. (2014) Intestinal inflammation and mucosal barrier function. Inflamm Bowel Dis 20, 2394-2404.

8. Anderson RC, Dalziel JE, Gopal PK, et al. (2012) The Role of Intestinal Barrier Function in Early Life in the Development of Colitis. Rijeka, Croatia: InTech.

9. Neish AS (2009) Microbes in gastrointestinal health and disease. Gastroenterology 136, 65-80.

10. Trachsel J, Briggs C, Gabler NK, et al. (2018) Resistant potato starch fuels beneficial host-microbe interactions in the gut. bioRxiv, 389007

11. Leonel AJ \& Alvarezleite JI (2012) Butyrate: implications for intestinal function. Curr Opin Clin Nutr Metab Care 15, 474-479.

12. Groschwitz K \& Hogan SP (2009) Intestinal barrier function: molecular regulation and disease pathogenesis. J Allergy Clin Immun 124, 3-20.

13. Keenan MJ, Zhou J, Hegsted M, et al. (2015) Role of resistant starch in improving gut health, adiposity, and insulin resistance. Adv Nutr 6, 198-205.

14. Sajilata MG, Singhal RS \& Kulkarni PR (2006) Resistant starch a review. Compr Rev Food Sci Food Saf 5, 1-17.

15. Nofrarías M, Martínez-Puig D, Pujols J, et al. (2007) Long-term intake of resistant starch improves colonic mucosal integrity and reduces gut apoptosis and blood immune cells. Nutrition 23, 861-870.

16. Fuentes-Zaragoza E, Riquelme-Navarrete MJ, Sánchez-Zapata E, et al. (2010) Resistant starch as functional ingredient: a review. Food Res Int 43, 931-942.

17. Jiminez JA, Uwiera TC, Abbott DW, et al. (2016) Impacts of resistant starch and wheat bran consumption on enteric inflammation in relation to colonic bacterial community structures and short-chain fatty acid concentrations in mice. Gut Pathog 8, 67.

18. Keenan MJ, Zhou J, Mccutcheon KL, et al. (2006) Effects of resistant starch, a non-digestible fermentable fiber, on reducing body fat. Obesity 14, 1523-1534.

19. Zhou J, Hegsted M, Mccutcheon KL, et al. (2006) Peptide YY and proglucagon mRNA expression patterns and regulation in the gut. Obesity 14, 683-689.

20. National Research Council (1994) Nutrient Requirements of Poultry, 9th rev. ed. Washington, DC: National Academies Press.

21. Nabuurs MJA, Hoogendoorn A, Der Molen EJV, et al. (1993) Villus height and crypt depth in weaned and unweaned pigs, reared under various circumstances in The Netherlands. Res Vet Sci 55, 78-84.

22. Ki Y, Kim W, Cho H, et al. (2014) The effect of probiotics for preventing radiation-induced morphological changes in intestinal mucosa of rats. J Korean Med Sci 29, 1372-1378.

23. Vandesompele J, De Preter K, Pattyn F, et al. (2002) Accurate normalization of real-time quantitative RT-PCR data by geometric averaging of multiple internal control genes. Genome Biol 3, RESEARCH0034.

24. Caporaso JG, Kuczynski J, Stombaugh J, et al. (2010) QIIME allows analysis of high-throughput community sequencing data. Nat Methods 7, 335-336.

25. Magoč T \& Salzberg SL (2011) FLASH: fast length adjustment of short reads to improve genome assemblies. Bioinformatics $\mathbf{2 7}$, $2957-2963$ 
26. Edgar RC, Haas BJ, Clemente JC, et al. (2011) UCHIME improves sensitivity and speed of chimera detection. Bioinformatics 27, 2194-2200.

27. Edgar RC (2013) UPARSE: highly accurate OTU sequences from microbial amplicon reads. Nat Methods 10, 996-998.

28. Quast C, Pruesse E, Yilmaz P, et al. (2013) The SILVA ribosomal RNA gene database project: improved data processing and web-based tools. Nucleic Acids Res 41, D590-D596.

29. Caporaso JG, Bittinger K, Bushman FD, et al. (2010) PyNAST: a flexible tool for aligning sequences to a template alignment. Bioinformatics 26, 266-267.

30. Price MN, Dehal PS \& Arkin AP (2010) FastTree 2-approximately maximum-likelihood trees for large alignments. PLOS ONE 5, e9490.

31. Oksanen J, Blanchet FG, Friendly M, et al. (2015) Vegan: Community Ecology Package. R Package Version. 2.3-1. https://cran.r-project.org/web/packages/vegan/

32. Whiteley LO, Higgins JM, Purdon MP, et al. (1996) Evaluation in rats of the dose-response relationship among colonic mucosal growth, colonic fermentation, and dietary fiber. Dig Dis Sci $\mathbf{4 1}$, $1458-1467$.

33. Fang L, Jiang X, Su Y, et al. (2014) Long-term intake of raw potato starch decreases back fat thickness and dressing percentage but has no effect on the longissimus muscle quality of growing-finishing pigs. Livest Sci 170, 116-123.

34. Wang JF, Zhu YH, Li DF, et al. (2004) In vitro fermentation of various fiber and starch sources by pig fecal inocula. J Anim Sci 82, 2615-2622.

35. Metzler-Zebeli BU, Canibe N, Montagne L, et al. (2018) Resistant starch reduces large intestinal $\mathrm{pH}$ and promotes fecal lactobacilli and bifidobacteria in pigs. Animal 13, 64-73.

36. Pourabedin $M \&$ Zhao X (2015) Prebiotics and gut microbiota in chickens. FEMS Microbiol Lett 362, fnv122

37. Vasaï F, Brugirard Ricaud K, Bernadet MD, et al. (2014) Overfeeding and genetics affect the composition of intestinal microbiota in Anas platyrhynchos (Pekin) and Cairina moschata (Muscovy) ducks. FEMS Microbiol Ecol 87, 204-216

38. Xiao Y, Xiang Y, Zhou W, et al. (2017) Microbial community mapping in intestinal tract of broiler chicken. Poult Sci 96 , 1387-1393.

39. Yacoubi N, Saulnier L, Bonnin E, et al. (2018) Short-chain arabinoxylans prepared from enzymatically treated wheat grain exert prebiotic effects during the broiler starter period. Poult $\mathrm{Sci}$ 97, 412-424.

40. Molist F, Manzanilla EG, Pérez JF, et al. (2011) Coarse, but not finely ground, dietary fibre increases intestinal Firmicutes: Bacteroidetes ratio and reduces diarrhoea induced by experimental infection in piglets. BrJ Nutr 108, 9-15.

41. Mulder IE, Schmidt B, Stokes CR, et al. (2009) Environmentallyacquired bacteria influence microbial diversity and natural innate immune responses at gut surfaces. BMC Biol 7, 79.

42. Biddle AS, Stewart LC, Blanchard JL, et al. (2013) Untangling the genetic basis of fibrolytic specialization by Lachnospiraceae and Ruminococcaceae in diverse gut communities. Diversity 5, 627-640.

43. Zeng H, Huang C, Lin S, et al. (2017) Lotus seed resistant starch regulates gut microbiota and increases SCFAs production and mineral absorption in mice. J Agric Food Chem $\mathbf{6 5}$, 9217-9225.

44. Zhang H, Dibaise JK, Zuccolo A, et al. (2009) Human gut microbiota in obesity and after gastric bypass. Proc Natl Acad Sci U S A 106, 2365-2370.

45. Zhang J, Guo Z, Xue Z, et al. (2015) A phylo-functional core of gut microbiota in healthy young Chinese cohorts across lifestyles, geography and ethnicities. ISME J 9, 1979-1990.
46. Sun Y, Su Y \& Zhu W (2016) Microbiome-metabolome responses in the cecum and colon of pig to a high resistant starch diet. Front Microbiol 7, 779

47. Martinez I, Kim J, Duffy PR, et al. (2010) Resistant starches types 2 and 4 have differential effects on the composition of the fecal microbiota in human subjects. PLOS ONE 5, e15046.

48. Walker AW, Ince J, Duncan SH, et al. (2011) Dominant and diet-responsive groups of bacteria within the human colonic microbiota. ISME J 5, 220-230.

49. Duncan SH, Louis P \& Flint HJ (2004) Lactate-utilizing bacteria, isolated from human feces, that produce butyrate as a major fermentation product. Appl Environ Microbiol 70, 5810-5817.

50. Gophna U, Konikoff T \& Nielsen HB (2017) Oscillospira and related bacteria - from metagenomics species to metabolic features. Environ Microbiol 19, 835-841.

51. Zhang L, Wu W, Lee YK, et al. (2018) Spatial heterogeneity and co-occurrence of mucosal and luminal microbiome across swine intestinal tract. Front Microbiol 9, 48.

52. Peng L, Li Z, Green RS, et al. (2009) Butyrate enhances the intestinal barrier by facilitating tight junction assembly via activation of amp-activated protein kinase in caco-2 cell monolayers. J Nutr 139, 1619-1625.

53. Vieira EL, Leonel AJ, Sad AP, et al. (2012) Oral administration of sodium butyrate attenuates inflammation and mucosal lesion in experimental acute ulcerative colitis. J Nutr Biochem 23, 430-436.

54. Johansson ME (2014) Mucus layers in inflammatory bowel disease. Inflamm Bowel Dis 20, 2124-2131.

55. Turner JR (2009) Intestinal mucosal barrier function in health and disease. Nat Rev Immunol 9, 799-809.

56. Wang HB, Wang PY, Xin W, et al. (2012) Butyrate enhances intestinal epithelial barrier function via up-regulation of tight junction protein claudin-1 transcription. Dig Dis Sci 57, 3126-3135.

57. Drucker DJ \& Yusta B (2014) Physiology and pharmacology of the enteroendocrine hormone glucagon-like peptide-2. Annu Rev Physiol 76, 561-583.

58. Tappenden KA, Albin DM, Bartholome AL, et al. (2003) Glucagon-like peptide- 2 and short-chain fatty acids: a new twist to an old story. J Nutr 133, 3717.

59. Hu XF, Guo YM, Huang BY, et al. (2010) The effect of glucagonlike peptide 2 injection on performance, small intestinal morphology, and nutrient transporter expression of stressed broiler chickens. Poult Sci 89, 1967-1974

60. Gil-Cardoso K, Comitato R, Gines I, et al. (2019) Protective effect of proanthocyanidins in a rat model of mild intestinal inflammation and impaired intestinal permeability induced by LPS. Mol Nutr Food Res 63, e1800720.

61. Amasheh M, Fromm A, Krug SM, et al. (2010) TNF $\alpha$-induced and berberine-antagonized tight junction barrier impairment via tyrosine kinase, Akt and NFKB signaling. J Cell Sci 123, 4145-4155.

62. Nathalie F, Cécile L, Pascale H, et al. (2014) Interleukin-6 receptor shedding is enhanced by interleukin-1beta and tumor necrosis factor alpha and is partially mediated by tumor necrosis factor alpha-converting enzyme in osteoblast-like cells. Arthritis Rheum 52, 84-93.

63. Qian Y, Zhao X, Song JL, et al. (2013) Inhibitory effects of resistant starch (RS3) as a carrier for stachyose on dextran sulfate sodiuminduced ulcerative colitis in C57BL/6 mice. Exp Ther Med 6, 1312-1316.

64. Zhou ZY, Packialakshmi B, Makkar SK, et al. (2014) Effect of butyrate on immune response of a chicken macrophage cell line. Vet Immunol Immunopathol 162, 24-32.

65. Toden S, Bird AR, Topping DL, et al. (2007) Dose-dependent reduction of dietary protein-induced colonocyte DNA damage by resistant starch in rats correlates more highly with caecal butyrate than with other short chain fatty acids. Cancer Biol Ther 6, 253-258. 